\title{
On the reconstruction of 3-uniform hypergraphs from step-two degree sequences
}

\author{
A. Frosini * $\quad$ G. Palma ${ }^{\dagger} \quad$ S. Rinaldi $\ddagger$
}

November 25, 2020

\begin{abstract}
A nonnegative integer sequence is $k$-graphic if it is the degree sequence of a $k$-uniform simple hypergraph. The problem of deciding whether a given sequence $V$ admits a 3-uniform simple hypergraph has recently been proved to be $N P$-complete, after long years of research. Thus, it is helpful to find which classes of instances are polynomially solvable in order to restrict the NP-hard core of the problem and design algorithms for real-life applications. Several necessary and few sufficient conditions for $V$ to be $k$-graphic, with $k \geq 3$, appear in the literature. Frosini et al. defined a polynomial-time algorithm to reconstruct $k$-uniform hypergraphs having regular or almost regular degree sequences. Our study fits in this this research line defining some conditions and a polynomial-time algorithm to reconstruct 3-uniform hypergraphs having step-two degree sequences, i.e. $V=(d, \ldots, d, d-2 \ldots, d-2)$. Our results are likely to be easily generalized to $k \geq 4$ and to other families of degree sequences having regular shapes.

KEYWORDS: 3-UNIFORM HYPERGRAPH, DEGREE SEQUENCE, DISCRETE TOMOGRAPHY, RECONSTRUCTION PROBLEM
\end{abstract}

\section{Introduction}

The degree sequence of a simple hypergraph is the list of its vertex degrees, usually arranged in decreasing order. Given a nonnegative integer sequence

\footnotetext{
${ }^{*}$ Università di Firenze, Dipartimento di Matematica e Informatica, Viale Morgagni 65, 50134 Firenze, Italy

${ }^{\dagger}$ Università di Siena, Dipartimento di Ingegneria dell'Informazione e Scienze Matematiche, Via Roma 56, 53100 Siena, Italy

${ }^{\ddagger}$ Università di Siena, Dipartimento di Ingegneria dell’Informazione e Scienze Matematiche, Via Roma 56, 53100 Siena, Italy
} 
$V$, the possibility of an efficient test for the existence of a simple hypergraph having degree sequence $V$ has remained unsolved for many years (see [2], [4]). The corresponding problem for graphs was solved by the Erdös-Gallai conditions ([8]). From their result, a polynomial time algorithm can be defined to reconstruct the adjacency matrix of a graph having a given degree sequence (if such a graph exists).

Assuming that $P \neq N P$, such an effective characterization cannot exist even for the case of 3-uniform hypergraphs (see Deza et. al [6]).

Necessary and sufficient conditions for a nonnegative integer sequence $V$ to be $k$-graphic, with $k \geq 3$, can be found in the literature, and they rely mainly on a result by Dewdney [5], based on a recursive decomposition of $V$. However this characterization does not yield an efficient algorithm and the question to determine a more practical characterization remained open. Brlek and Frosini in [3] defined a $P$-time algorithm in case of homogenous degree sequences. Later this result was extended to almost regular sequences. A remarkable fact is that in both cases, all the 3-graphic sequences satisfy a simple necessary and sufficient condition. In our work, we investigate steptwo 3-graphic sequences and we discovered that such a conditions are not sufficient any more. So the step change from 1 to 2 in the degree sequence is critical for the reconstruction of the related 3-hypergraph.

Frosini, Picouleau and Rinaldi provided sufficient and necessary conditions in case of $k$-uniform and (almost) regular hypergraphs (see [9]). In our present study we we extend their result to two-step 3-graphic sequences. We will characterize them and prove some fundamental properties. Thus, relying on these results, we design an efficient algorithm that determines the incidence matrix of the characterized sequences.

In the next section, we provide the definitions and the previous results useful for our study, then, we introduce the main problem. The section 3 is devoted to the consistency problem concerning 3-uniform hypergraphs having two-step degree sequences. Then in section 4 , the reconstruction problem is solved and the related polynomial time algorithm for the 3-uniform hypergraphs is provided. We conclude the article pointing out some open questions concerning $k$-uniform hypergraphs and degree sequences having span two, in section 5 . 


\section{Definitions, previous results and introduction of the problems}

A hypergraph $\mathcal{H}$ is defined as a couple $(V e r, E)$, where $V e r$ is a finite set of vertices, $v_{1}, \ldots, v_{n}$, and $E$ is a set of hyperedges, i.e., a collection of subsets of $V e r,\left\{e_{1}, e_{2}, \ldots, e_{m}\right\}$ where each $e_{i}$ is a non-empty subset of $V e r$, (see [2]). A hypergraph is simple if it is loopless and without parallel hyperedges, i.e. $e \neq e^{\prime}$ for any pair $e, e^{\prime}$ of hyperedges. Moreover, a hypergraph is said to be $k$-uniform if each hyperedge has cardinality $k$. The degree of a vertex $v \in V e r$ is the number of hyperedges $e \in E$ such that $v \in e$. The degree sequence of $\mathcal{H}$ is the list of its vertex degrees, usually written in nonincreasing order. In this context, the problem of the characterization of the degree sequence $V=\left(d_{1}, d_{2}, \ldots, d_{n}\right)$ of a $k$-uniform hypergraph $\mathcal{H}$ asks whether there is a binary matrix $A \in \mathcal{U}(H, V)$ with nonnegative projections vectors $H=(k, k, \ldots, k)$ and $V=\left(d_{1}, d_{2}, \ldots, d_{n}\right)$ and distinct rows. The matrix $A$ is an incidence matrix of $\mathcal{H}$; thus, rows and columns correspond to hyperedges and vertices, respectively.

Let $H=\left(h_{1}, h_{2}, \ldots, h_{m}\right)$ and $V=\left(v_{1}, v_{2}, \ldots, v_{n}\right)$ be two nonincreasing vectors of nonnegative integers, and $\mathcal{U}(H, V)$ the class of binary matrices $A=\left(a_{i j}\right)$ satisfying $\sum_{j=1}^{n} a_{i j}=h_{i}$, with $1 \leq i \leq m$, and $\sum_{i=1}^{m} a_{i j}=$ $v_{j}$, with $1 \leq j \leq n$. In this context, the problem of the characterization of the degree sequence $\left(d_{1}, d_{2}, \ldots, d_{n}\right)$ of a $k$-uniform hypergraph $\mathcal{H}$ asks whether there is a binary matrix $A \in \mathcal{U}(H, V)$, its incidence matrix, with nonnegative projections vectors $H=(k, k, \ldots, k)$ and $V=\left(d_{1}, d_{2}, \ldots, d_{n}\right)$ and distinct rows. Rows and columns of $A$ correspond to hyperedges and vertices, respectively.

The investigation in the special case where the $k$-uniform hypergraph to reconstruct is also $d$-regular, i.e. each vertex $w$ has the same degree $d$, $V=(d, \ldots, d)$, or almost $d$-regular, i.e. $V=(d, \ldots, d, d-1, \ldots, d-1)$, was done by Frosini, Picouleau and Rinaldi in [9]. The authors studied the decision problem and gave the following conditions that characterize the degree sequences of $d$-regular hypergraphs: Condition 1: For each $1 \leq i \leq m$ and $1 \leq j \leq n$, it holds $h_{i} \leq n$ and $v_{j} \leq m$.

Condition 2: The sum of the entries of the horizontal and vertical projections are equal (i.e. $\sum_{i=1}^{m} h_{i}=\sum_{j=1}^{n} v_{j}$ ).

Condition 3: The following inequality holds: $d \leq \frac{k}{n} \cdot\left(\begin{array}{l}n \\ k\end{array}\right)$.

Moreover, they also analyzed the related reconstruction problem, i.e. the problem of determining an element of $\mathcal{U}(H, V)$ consistent with $H$ and 
$V$. To accomplish this task they design a polynomial-time algorithm, with respect to the dimensions $m$ and $n$ of the matrix to reconstruct. Since each row of a binary solution matrix can be regarded as a binary finite word $u=u_{1} u_{2} \ldots u_{n}$, whose length $n$ is the number of columns of the matrix, and whose number of 1-elements is the common horizontal projection, that is 3; Frosini at al. used Lyndon words and necklaces of fixed density, in their algorithm. Following the notation in [13], we recall that a binary necklace is the equivalence class of binary words under cyclic shift. In particular, an aperiodic word is called a Lyndon word. We are interested in fixed-density words (resp. Lyndon words), in which an additional parameter $h$, called density, that represents the number of $1 \mathrm{~s}$ in the words, is added. The set of necklaces (resp. Lyndon words) with density $h$ is represented by $N(n, h)$ (resp. $L(n, h))$. Concerning step-two nonnegative integer sequences, i.e. $V=(d, \ldots, d, d-2, \ldots, d-2)$, it can be easily observed that there are instances (e.g. $V=(7,7,7,7,7,7,5,5,5))$ that satisfy Conditions 1,2 and 3 , but the same method of reconstruction of $d$-regular hypergraphs cannot be applied. In some cases, however, the matrix can still be reconstructed.

In light of this, we present results concerning step-two sequences in the special case of 3 -uniform hypergraphs. For sake of brevity, we denote $\mathcal{P}$, the set of all step-two integer sequences that satisfy Conditions 1, 2, and 3 , and writing vectors we sometimes use the exponential notation, i.e. $\left(s^{x}, f^{y}\right)$ indicates a vector with $x$ times the component $\mathrm{s}$, followed by $y$ times $\mathrm{f}$.

We believe these few notions are enough to state the two problems we will address in this paper.

\section{$\operatorname{Consistency}(V, \mathcal{C})$}

Input: an integer vector $V=(d, \ldots, d, d-2, \ldots, d-2)$, and a class of discrete sets $\mathcal{C}$.

Question: does there exist an element of $\mathcal{C}$ whose horizontal and vertical projections are the homogenous vector $H=(3, \ldots 3)$ and $V$, respectively?

\section{Reconstruction $(V, \mathcal{C})$}

Input: an integer vector $V=(d, \ldots, d, d-2, \ldots, d-2)$, and a class $\mathcal{C}$ of discrete sets.

Task: reconstruct a matrix $A \in \mathcal{C}$ whose horizontal and vertical projections are the homogenous vector $H=(3, \ldots 3)$ and $V$, respectively, if it exists, otherwise give failure.

In the sequel we are going to consider the class of binary matrices having no equal rows and homogeneous horizontal projections $H=(3, \ldots, 3)$ of length 
$m$ and step-two vertical projection $V$, denoted $\mathcal{E}$, due to its connections with the characterization of the degree sequences of 3-uniform hypergraphs.

\section{The Problem Consistency $(V, \mathcal{E})$}

We face the consistency problem for the class $\mathcal{E}$ on the instance $V=$ $(d, \ldots, d, d-2, \ldots, d-2)$.

We now give the definition of the complement of a step-two degree sequence, as it will be useful in the following.

Given a step-two degree sequence $V=\left(d^{g},(d-2)^{n-g}\right)$, we define complement of $V$, denoted by $\bar{V}$, the vector $\bar{V}=\left(\left(d_{\max }-d+2\right)^{n-g},\left(d_{\max }-d\right)^{g}\right)$, where $d_{\max }=\frac{k}{n} \cdot\left(\begin{array}{l}n \\ k\end{array}\right)$.

A direct consequence of the definition of complement of a degree sequence is the following proposition:

Proposition 1. Given a degree sequence $V$, if $V$ is $k$-graphic then also its complement $\bar{V}$ is k-graphic.

Proof. The fact that $V$ is $k$-graphic means that we can reconstruct the incidence matrix associated with $V$. Given the incidence matrix associated with the homogeneous regular vector $(d, d, \ldots, d)$ of length $n$ (the latter matrix can be reconstructed, see [9]), the rows that remain from the elimination of a set of rows whose vertical projection is the vector $V$, have exactly $\bar{V}$ read from right to left as vector of vertical projections. It is a direct consequence of the definition of complement of $V$. By flipping horizontally the columns of the obtained matrix, we find the incidence matrix of a hypergraph having $\bar{V}$ as degree sequence. Hence, $\bar{V}$ is $k$-graphic.

There are no 3 -uniform hypergraphs having step-two degree sequences of length $n \leq 4$. Concerning step-two sequences in $\mathcal{P}$, whose length is $n \geq 5$, we show the following result:

Theorem 2. Every step-two sequence $V=\left(2^{3}, 0^{p}\right)$, with $p \geq 1$, is not a 3-graphic sequence.

Proof. The matrix associated with the sequence $V=\left(2^{3}, 0^{p}\right)$ is not reconstructable, because it would need two equal rows.

Let us call basic sequences, the integer sequences $\left(d^{g},(d-2)^{n-g}\right) \in \mathcal{P}$, with $2 \leq d \leq 4$, not considered in Theorem 2 and the sequences $\left(5^{3}, 3^{p}\right)$, with $p>0$. 
Proposition 3. Every basic sequence is a 3-graphic sequence.

Proof. We obtain the result by defining, for each type of basic sequences a class of 3-hypergraphs satisfying it. We consider separately the cases of the basic sequences:

1. $\left(2^{3 g}, 0^{p}\right)$, with $g>1, p \geq 1$ and such that $3 g+p>4$;

2. $\left(3^{g}, 1^{3 p}\right)$, with $g \geq 1, p \geq 1$ and such that $g+3 p>4$;

3. $\left(4^{3 g}, 2^{3 p}\right)$, with $g \geq 1, p \geq 1$ and such that $3 g+3 p>4$;

4. $\left(5^{3}, 3^{p}\right)$, with $p \geq 3$.

Case 1 can be reconstructed just using the cyclic shifts of the Lyndon word $(111)(0)^{n-3}$ where the first 1-element is in position $i$, with $1 \leq i<$ $3 g-1$ and $i$ is not a multiple of 3 ; and the Lyndon word $10^{3(g-1)} 110^{n-3 g}$.

Case 2 is divided into 3 subcases:

2.1. If $g=1$, then $p \geq 2$ (otherwise $n=4$ ). We use the Lyndon word $1^{3} 0^{n-3}$ and its cyclic shift $10^{n-3} 11$. We eventually use the cyclic shifts $0^{5} 1110^{n-8}, \ldots, 0^{n-5} 11100$, if necessary. Then we use the Lyndon word $100110^{n-5}$.

2.2. If $g=2$; we use the Lyndon word $1^{3} 0^{n-3}$ and its cyclic shift $110^{n-3} 1$. We eventually use the cyclic shifts $0^{4} 1110^{n-7}, \ldots, 0^{n-4} 1110$, if necessary. Then we use the Lyndon word $11010^{n-4}$.

2.3. If $g \geq 3$ we use the Lyndon word $1^{3} 0^{n-3}$ and its cyclic shifts, where the first 1-element is in position $i$, with $1 \leq i \leq g-1$; thus, its cyclic shift $110^{n-3} 1$. We eventually use the cyclic shifts $0^{g+2} 1110^{n-g-5}, \ldots$, $0^{n-4} 1110$, if necessary. Then we use the Lyndon word $10^{g-2} 1010^{n-g-2}$.

Case 3 is divided into 3 subcases:

3.1. $\left(4^{3 x}, 2^{3 y}\right)$, with $x, y>0, n>6$, since the length is a multiple of 3 , we can use the cyclic shifts of the periodic word $\left(10^{n / 3-1}\right)^{3}$, and the matrix associated with the sequence $\left(3^{3 x}, 1^{3 y}\right)$ that we have already reconstructed in Case 2.

3.2. $\left(4^{3 x+1}, 2^{3 y+1}\right)$, with $x, y \geq 0$, we use the cyclic shifts of the Lyndon word $(111) 0^{n-3}$, with the first 1 -element in position $i$, with $1 \leq i \leq 3 x$; the cyclic shifts of the same Lyndon word with the first 1-element in position $3 x+3, \ldots, n-2$. Now, we have to reconstruct the matrix 
associated with the sequence $S=\left(2^{2}, 1^{3 x-1}, 0^{3 y+1}\right)$. This can be done wisely arranging the cyclic shifts of the the Lyndon word 10101(0) ${ }^{n-5}$. If the length of the sequence $S$ is even, this is sufficient. Instead, we have also to use the Lyndon word $110^{n-3} 1$.

3.3. $\left(4^{3 x+2}, 2^{3 y+2}\right)$, with $x, y \geq 0$, we use the cyclic shifts of the Lyndon word (111) $0^{n-3}$, with the first 1 -element in position $i$, with $1 \leq i \leq$ $3 x+2$; the cyclic shift of the same Lyndon word with the first 1-element in position $3 x+4, \ldots, n-1$. Now, we have to reconstruct the matrix associated with the sequence $S=\left(2,1^{3 x+1}, 0^{3 y+2}\right)$. Analogously as before, this can be done using three Lyndon words at most.

Case 4 can be reconstructed using the matrix obtained from the matrix of the necklaces obtained by cling shifting the word $1^{3} 0^{n-3}$, and removing the necklace $0^{n-3} 1^{3}$. Now, we have to reconstruct the matrix associated with the sequence $G=\left(2^{3}, 0^{n-6}, 1^{3}\right)$. This can be done using the Lyndon word $110^{n-5} 10^{2}$ and its cyclic shift $0110^{n-5} 10$, and the Lyndon word $1010^{n-4} 1$.

Remark 4. The basic sequences can be reconstructed using three different necklaces at most.

The procedure that reconstructs the incidence matrix associated with a basic element $V$ is denoted $\operatorname{RecBasic}(V)$.

\section{An Algorithm to Solve Reconstruction $(V, \mathcal{E})$}

We recall that Sawada [15] presented a constant amortized time (CAT) algorithm FastFixedContent for the exhaustive generation of necklaces $N(n, h)$ of fixed length and density, and a slight modification of it, here denoted GenLyndon $(n, h)$, for the CAT generation of the Lyndon words $L(n, h)$. This latter constructs a generating tree of the words, and since the tree has height $h$, the computational cost of generating $k$ words of $L(n, h)$ is $O(k \cdot h \cdot n)$.

Our reconstruction algorithm works as depicted in Algorithm 1.

Proposition 5. For $n>4$ there is a sufficient number of Lyndon words required in the application of the Algorithm 1.

Proof. For $n=5$ there are only four sequences in $\mathcal{P}: V_{1}=\left(3^{2}, 1^{3}\right)$ and its complement $\overline{V_{1}}=\left(5^{3}, 3^{2}\right)$, and $V_{2}=\left(4,2^{4}\right)$ and its complement $\overline{V_{2}}=\left(4^{4}, 2\right)$. $V_{1}$ is basic sequences. Since $L(5,3)=2$ and the procedure $\operatorname{RecBasic}\left(V_{1}\right)$ 
$\operatorname{Algorithm~} 1 \operatorname{Rec}(V, \mathcal{E})$

Input: The vector $V=(d, \ldots, d, d-2, \ldots, d-2) \in \mathcal{P}$ of length $n \geq 5$; and the class $\mathcal{E}$.

Output: An element of the class $\mathcal{E}$.

We solve $\operatorname{Rec}\left(V_{i}, \mathcal{E}\right)$, where $V_{i}$ is the minimum between $V$ and $\bar{V}$ in the lexicographical order .

Step 1: We initialize the matrix $B=\emptyset$, and the vector $D=V$. By applying $\operatorname{GenLyndon}(n, 3)$, generate the sequence of Lyndon words $u_{1}, \ldots, u_{q}$.

Step 2: While $D$ is not a basic sequence, do $D=D-\left(3^{n}\right)$.

Step 3: $B=\operatorname{RecBasic}(D)$.

Step 4: While $D$ is different from $V$, generate the matrix $M\left(u_{i}\right)$, where $u_{i}$ is a word not already taken in $B$. Add $M\left(u_{i}\right)$ to $B$ and update the vector of the vertical projection $D$.

requires just two words then they are sufficient to reconstruct the matrices associated with $V_{1}$. Concerning $V_{2}$, the procedure $\operatorname{RecBasic}\left(V_{2}\right)$ would need three words, however, as the following matrix shows, two words are sufficient.

$$
\left[\begin{array}{lllll}
1 & 1 & 1 & 0 & 0 \\
1 & 0 & 0 & 1 & 1 \\
\hline 1 & 1 & 0 & 1 & 0 \\
1 & 0 & 1 & 1 & 0
\end{array}\right]
$$

We observe that the sequence $\overline{V_{1}}$ is not a basic sequence and does not fall within the sequences considered in Theorem 2. However, it can be reconstructed as a complement to a 3 -graphic sequence.

For $n=6$ there are only seven sequences in $\mathcal{P}: V_{1}=\left(3^{3}, 1^{3}\right)$ and its complement $\overline{V_{1}}=\left(9^{3}, 7^{3}\right), V_{2}=\left(4^{3}, 2^{3}\right)$ and its complement $\overline{V_{2}}=\left(8^{3}, 6^{3}\right)$, $V_{3}=\left(5^{3}, 3^{3}\right)$ and its complement $\overline{V_{3}}=\left(7^{3}, 5^{3}\right)$, and $V_{4}=\overline{V_{4}}=\left(6^{3}, 4^{3}\right)$. Sequences $V_{1}, V_{2}, V_{3}$ are basic sequences and can be reconstructed with a maximum of 3 Lyndon words. Since $L(6,3)=4$, the words are sufficient. Concerning $V_{4}$ four words are sufficient too, indeed it takes two words to apply the procedure $\operatorname{RecBasic}\left(V_{1}\right)$, and then another word to obtain a matrix having vertical projections $(3,3,3,3,3,3)$.

For $n>6$, there is a sufficient number of Lyndon words as the following inequality holds:

$$
\frac{\left\lceil\frac{1}{2} \cdot \frac{3}{n}\left(\begin{array}{c}
n \\
3
\end{array}\right)\right\rceil-d_{B}}{3} \geq 3
$$

where $\left\lceil\frac{1}{2} \cdot \frac{3}{n}\left(\begin{array}{l}n \\ k\end{array}\right)\right\rceil$ is the maximum degree that can appear in a step-two degree 
sequence in $\mathcal{P}$ (except the complements of sequences that preced them in the lexicographical order), $d_{B}$ is the greatest degree of a basic sequence (hence $d_{B}=2$, or $d_{B}=3$, or $d_{B}=3$ or $d_{B}=4$, or $d_{B}=5$ ) and $n$ is the length of the degree sequence. From the previous inequality we get the worst case behavior:

$$
(n-1) \cdot(n-2) \geq 4 \cdot\left(9+d_{B}\right)
$$

Theorem 6. Given a sequence $V \in \mathcal{P}, V$ is 3-graphical if and only if $V$ can be reconstructed with the Algorithm 1.

Proof. $(\Leftarrow)$ is trivial.

$(\Rightarrow)$ Suppose that $V$ is a 3-graphic sequence and that it cannot be reconstructed with the Algorithm 1. Then there are two possibilieties:

1. There are not enough words to complete the algorithm.

2. The procedure RecBasic can not be applied, since one of the sequences considered in Theorem 2.

Case 1 cannot occur because Proposition 5 proves that there are always a sufficient number of words. Concerning Case 2, since there is a sufficient number of Lyndon words, we reach a sequence in which the maximum degree is less than or equal to 5 and is considered in the Theorem 2 , that is $B_{1}=$ $\left(2^{3}, 0^{p}\right)$, with $p \geq 1$. It is not possible that the algorithm takes as input $V$ which is 3 -graphical and has to reconstruct $B_{1}$, since for such $V$ the algorithm would stop at the basic sequence $\left(5^{3}, 3^{p}\right)$.

To better understand the reconstruction algorithm, we propose a simple example with the instance $H=(3, \ldots, 3)$ of length $m=15$, and $V=(7,7,7,7,7,5,5)$ of length $n=7$. In Step 1 the matrix $B$ is initialized as the empty matrix, and the vector $D=(7,7,7,7,7,5,5)$. Then, Gen$\operatorname{Lyndon}(7,3)$ generates $q=5$ Lyndon words, i.e. the words $u_{1}=0000111$, $u_{2}=0001011, u_{3}=0001101, u_{4}=0010011$, and $u_{5}=0010101$. From Step 2 , we get $D=(4,4,4,4,4,2,2)$. In Step 3 , using the procedure RecBasic we reconstruct the matrix $B$ associated with $D$. In Step 4 , we add the matrix $M\left(u_{2}\right)$ to $B$. We evaluate the vector of the vertical projection $D$. Since $D$ is equal to $V$, we stop. The output matrix $C$, depicted in Figure 1. 


$$
\left[\begin{array}{lllllll}
0 & 0 & 0 & 1 & 0 & 1 & 1 \\
1 & 0 & 0 & 0 & 1 & 0 & 1 \\
1 & 1 & 0 & 0 & 0 & 1 & 0 \\
0 & 1 & 1 & 0 & 0 & 0 & 1 \\
1 & 0 & 1 & 1 & 0 & 0 & 0 \\
0 & 1 & 0 & 1 & 1 & 0 & 0 \\
0 & 0 & 1 & 0 & 1 & 1 & 0 \\
\hline 1 & 1 & 1 & 0 & 0 & 0 & 0 \\
0 & 1 & 1 & 1 & 0 & 0 & 0 \\
0 & 0 & 1 & 1 & 1 & 0 & 0 \\
0 & 0 & 0 & 1 & 1 & 1 & 0 \\
0 & 0 & 0 & 0 & 1 & 1 & 1 \\
1 & 1 & 0 & 0 & 0 & 0 & 1 \\
\hline 1 & 0 & 1 & 0 & 1 & 0 & 0 \\
\hline 1 & 1 & 0 & 1 & 0 & 0 & 0
\end{array}\right]
$$

The validity of $\operatorname{Rec}(V, \mathcal{E})$ is a simple consequence of Theorem 6 . Clearly, the obtained matrix has homogeneous horizontal projection equal to $H$, and step-two vertical projection equal to $V$, and, by construction, all the rows are distinct. Moreover, the algorithm always terminates since at each iteration, we add as many rows as possible to the final solution. Concerning the complexity analysis, we need to generate $O(m)$ different Lyndon words and shifts each of them $O(n)$ times. Thus, since the algorithm GenLyn$\operatorname{don}(n, 3)$ requires $O(f \cdot h \cdot n)$, that is $O(3 \cdot f \cdot n)$ steps to generate $f$ words of $L(n, 3)$, the whole process takes polynomial time.

\section{Conclusions and future developments}

We designed a polynomial algorithm that reconstructs the incidence matrix of a 3-uniform hypergraph realizing a step-two sequence of integers, when such a hypergraph exists.

The algorithm we furnished, is tuned for 3-uniform hypergraphs, but we believe that it admits a generalization to $k$-uniform ones. Thus, a possible direction for further research is to generalize our algorithm to obtain a polynomial algorithm for the reconstruction of $k$-uniform hyeprgraphs having a step-two degree sequence. The characterization of the degree sequences of $k$-uniform hyperpgraphs, $k \geq 3$, is an $N P$-hard problem. Therefore, under the assumption that $P \neq N P$ there is no hope to find a good characterization; but to find a compact nice looking characterization should be of great interest in order to design algorithms for real-life applications. 
Another line of research concerns the characterization and the study of integer sequences having span two, i.e. $V=(d, \ldots, d, d-1, \ldots, d-1, d-$ $2, \ldots, d-2)$.

\section{References}

[1] Behrens, S., Erbes, C., Ferrara, M., Hartke, S.G., Reiniger, B., Spinoza, H., Tomlinson, C.:New Results on Degree Sequences of Uniform Hypergraphs, Electron. J. Comb., 20(4), \# P14 (2013).

[2] C. Berge, Hyperpgraphs, North-Holland, Amsterdam, (1989).

[3] Brlek, S., Frosini, A.: A Tomographical Interpretation of a Sufficient Condition for h-Graphical Sequences DGCI 2016, Lect. Notes in Comput. Sc., 9647, pp. $95-104$ (2016).

[4] C.J. Colbourn, W.L. Kocay, D.R. Stinson, Some NP-complete problems for hypergraph degree sequences,Discrete Applied Maths. 14 (1986), 239254 .

[5] Dewdney, A.K., Degree sequences in complexes and hypergraphs, Proc. Amer. Math. Soc., 53(2), pp. 535 - 540 (1975).

[6] A. Deza, A. Levin, S.M. Meesum, S. Onn, Optimization over degree sequences, SIAM J. Fisc. Math. Vol 32 (3), 2067-2079 (2018).

[7] Douglas B. West, Introduction to Graph Theory, Prentice Hall, Upper Saddle River, NJ, (2001).

[8] P. Erdös, T. Gallai, Graphs with prescribed degrees of vertices (Hungarian), Mat. Lapok, Vol. 11, 264-274 (1960).

[9] A. Frosini, C. Picouleau, S. Rinaldi, On the degree sequences of uniform hypergraphs, Discrete Geometry for Computer Imagery, Springer, (2013), 300-310.

[10] E. N. Gilbert, J. Riordan, Symmetry types of periodic sequences, Illinois J. Math. Vol. 5, Issue 4 (1961), 657-665.

[11] Herman, G.T., Kuba, A. (Eds.): Discrete tomography: Foundations algorithms and applications, Birkhauser, Boston (1999).

[12] Herman, G.T., Kuba, A. (Eds.): Advances in Discrete Tomography and its Applications, Birkhauser, Boston (2006). 
[13] F. Ruskey, J. Sawada, An efficient algorithm for generating necklaces with fixed density, Siam J. Comput. 29, 671-684 (1999).

[14] H.J. Ryser, Combinatorial Mathematics, Mathematical Association of America and Quinn and Boden, Rahway, New Jersey (1963).

[15] J. Sawada, A fast algorithm to generate necklaces with fixed content, Theoretical Computer Science 301 (2003) 477-489. 